\title{
Factores estresantes asociados al hiperestrés en estudiantes universitarios de la ciudad de Chilpancingo, Guerrero, México
}

\section{Stressors associated with hiperstress in undergraduate students in the city of Chilpancingo, Guerrero, Mexico}

\author{
Mireya Maruris Reducindo ${ }^{1} \quad$ Pedro Cortés Genchi \\ Miguel Ángel Cabañas Guerrero Flaviano Godínez Jaimes \\ Universidad Autónoma de Guerrero, México \\ Mónica González Ramírez René Landero Hernández \\ Universidad Autónoma de Nueva León, México
}

(Rec: abril de 2012 - Acep: junio de 2012)

\begin{abstract}
Resumen
El objetivo fue conocer el nivel de estrés y los factores asociados en estudiantes de licenciatura de las diferentes Unidades Académicas de la Ciudad Universitaria de Chilpancingo, Guerrero, México. Corresponde a un estudio de tipo transversal, realizado en una muestra de 500 estudiantes de 17 a 44 años, la edad promedio fue de $21.29 \pm 3.13$ años. La selección de estudiantes fue al azar. La medición del nivel de estrés se realizó con un estresómetro que contiene 96 preguntas relacionadas con el estilo de vida, ambiente, síntomas, empleo/ ocupación, relaciones y personalidad. La prevalencia de hiperestrés fue de 44.4\%. Las Unidades Académicas con mayor prevalencia de estrés fueron Ciencias Químicas (56\%) y Filosofía y Letras (52.54\%). Las mujeres tienen mayor prevalencia en estrés que los hombres. Se encontraron 17 factores asociados al hiperestrés, entre los que se encuentran: no hacer ejercicio, consumo de alcohol y sentirse cansado y sin energías, entre otros.

Palabras clave: prevalencia estrés, estrés, estresores y estudiantes.
\end{abstract}

\begin{abstract}
The objective was to determine the level of stress and associated factors in undergraduates students from all the Academic Units located in the University City in Chilpancingo, Gro. A cross-sectional study was conducted in a random sample of 500 students with ages ranging from 17-44 years, the average age was $21.29 \pm 3.13$ years. Stress levels were measured with an estresometer containing 96 questions related to lifestyle, environment, symptoms, employment/occupation, relationships and personality. The prevalence of hiperestrés was $44.4 \%$. The Academic Units with more prevalence of stress were Chemistry Sciences (56\%) and Philosophy and Literature (52.54\%). The women have more stress that the men. We found 17 factors associated with hiperstress, among which are: no exercise, alcohol consumption, feeling tired and without energy, among others.
\end{abstract}

Keywords: prevalence stress, stress, stressors and students.

\footnotetext{
1 Correspondencia dirigida a: Mireya Maruris Reducindo. Universidad Autónoma de Guerrero, Unidad Académica de Ciencias Naturales. Carretera Nacional, Chilpancingo-Petaquillas, Ex rancho El Shalako, Chilpancingo, Guerrero, México. Tel. 01 (747) 4729235 y 01 (747) 47292 35; Celular 7471104723. E-mail: maruris16@hotmail.com.
} 


\section{Introducción}

Actualmente el estrés es un tema común en investigaciones psicológicas, fisiológicas y laborales, debido a las condiciones ambientales, sociales, personales y económicas a las que el individuo se enfrenta cotidianamente (Hernández, Cerezo \& López, 2007). El estrés es el comienzo de una serie de enfermedades $y$, aunque no es la causa directa de las mismas, con frecuencia contribuye a su desarrollo. No es necesariamente nocivo, ya que a menudo ayuda a alcanzar los objetivos deseados (Melgosa, 2006).

El estrés se define como un conjunto de reacciones fisiológicas y psicológicas que experimenta el organismo cuando es sometido a fuertes demandas. Cuando estamos en un estado de estrés, el organismo se prepara para el ataque o huida (Melgosa, 2006).

La presencia del estrés en estudiantes de todos los niveles y edades es una realidad que acontece en las instituciones académicas (Barraza, 2004). Se ha reportado que en las licenciaturas del área de la Salud los estudiantes manifiestan mayores niveles de estrés, según Carmel y Bernstein (1987). De hecho, la escuela de Medicina presenta un ambiente muy estresante; y se ha evidenciado que los estudiantes con menor nivel de estrés presentan un mejor resultado académico (Román, Ortiz \& Hernández, 2008).

Polo, Hernández y Pozo (1996) estudiaron cómo el estilo de vida de los estudiantes se ve modificado según se acerca el periodo de exámenes, convirtiéndose así sus hábitos en insalubres, como exceso en el consumo de cafeína, tabaco, sustancias psicoactivas como excitantes y en algunos casos uso de tranquilizantes, lo que posteriormente puede llevar a la aparición de trastornos en la salud.

En el estado de Guerrero no se tienen registros de la prevalencia del hiperestrés en estudiantes universitarios, por lo que fue necesario realizar la presente investigación para conocer los niveles de estrés y los factores asociados al hiperestrés y qué licenciaturas y semestres presentan las mayores prevalencias.

\section{Método}

Se realizó un estudio transversal en una población de estudiantes universitarios en la ciudad de Chilpancingo, Guerrero, de los diferentes programas de Licenciatura que se imparten en las Unidades Académicas de la Universidad Autónoma de Guerrero, ubicadas en Ciudad Universitaria: Ciencias Químico Biológicas, Derecho, Economía, Filosofía y Letras,
Ingeniería y Matemáticas. El grupo de estudio fueron alumnos de $1^{\circ}, 3^{\circ}, 5^{\circ}, 7^{\circ}$ y $9^{\circ}$ semestre de cada programa de Licenciatura.

El tamaño de la población de acuerdo con listas del total de alumnos inscritos del presente ciclo escolar, proporcionado por la Dirección de Administración Escolar y Certificación de Competencias, fue de 6784 estudiantes.

Se realizó un muestreo estratificado, considerando cada grado como estrato y en éste se realizó un muestreo al azar para elegir los grupos y en cada grupo se seleccionó al azar a los estudiantes que participaron en el estudio. El tamaño de la muestra se calculó con un 95\% de confianza y una proporción de 0.30 (Gómez, 2009).

El tamaño de la muestra estimada fue de 500 estudiantes, éstos se distribuyeron de manera proporcional de acuerdo al total de alumnos por cada escuela y grado. Por ejemplo, Ciencias Químicas tiene un total de 1110 alumnos con un $16 \%$ del total, por lo que le corresponden 80 alumnos a encuestar.

Estos 80 alumnos fueron distribuidos de forma aleatoria en las dos licenciaturas con las que cuenta esta Unidad Académica (Químico Biólogo Parasitólogo y Biólogo), de igual manera en ambos turnos y en los semestres.

\section{Procedimiento}

La medición del nivel de estrés se realizó mediante la aplicación de un estresómetro, que consta de 96 preguntas en las cuales se evaluó: el estilo de vida, ambiente, síntomas, empleo/ocupación, relaciones y personalidad. También se tomaron medidas antropométricas como el peso, talla, índice de cintura cadera. Con el baumanómetro Citizen $\mathrm{CH}-432 \mathrm{~B}$ se midió la presión arterial. Los alumnos que aceptaron participar firmaron una carta de consentimiento y se les informó que los datos proporcionados son confidenciales.

El diagnóstico se entregó inmediatamente. De acuerdo al puntaje obtenido en el cuestionario se determinaron 5 niveles de estrés. Zona 1, nivel de estrés peligrosamente bajo con menos de 48 puntos; zona 2 , estrés bajo de 48 a menos de 72 puntos; zona 3 , estrés normal de 72-118 puntos; zona 4, estrés elevado de 119-144 puntos y zona 5, estrés peligroso de 145 puntos o más (Melgosa, 2006).

A todos los estudiantes se les entregaron recomendaciones sobre dieta y ejercicios y a los que presentaron hiperestrés (estrés elevado y peligroso) se les entregaron las recomendaciones antes mencionadas y un plan de 5 días para disminuir el nivel de estrés.

Antes de aplicar la encuesta se realizó una prueba piloto para probar el instrumento de medición (estresómetro), 
en una población de 20 personas con características similares a la población de estudio. Una vez aplicada la prueba se procedió a modificar y precisar las preguntas para conformar el instrumento final. El estresómetro también fue utilizado por Maruris, Cortés, Gómez y Godínez (2011), para medir el nivel de estrés en una población de jóvenes y adultos de Chilpancingo, Guerrero. El alfa de Cronbach total que obtuvieron fue de 0.915 , lo cual refleja un buen nivel de consistencia interna.

Una vez recolectados los datos se realizó la captura en Epi data y el análisis estadístico en el paquete Stata versión 8.0. Primeramente se hizo una exploración de la base de datos, describiendo los porcentajes, la media y desviación estándar; se llevó a cabo un análisis bivariado y multivariado, tomando como variable dependiente el diagnóstico de hiperestrés definido como tener estrés elevado o estrés peligroso y como variables independientes todos los factores estresantes.

Se ocuparon tablas de contingencia de $2 \times 2$, que permitió calcular la magnitud del efecto a través de la razón de momios y los intervalos de confianza al 95\%. Se realizó el modelaje estadístico y las variables que individualmente mostraron asociación con el hiperestrés $(\mathrm{p}<0.05)$ se incluyeron en el Modelo de Regresión Logística, para ver qué variables de manera conjunta están asociadas al hiperestrés.

\section{Resultados}

\section{Análisis descriptivo}

Los estudiantes que participaron por cada Unidad Académica fueron los siguientes: 16\% (80/500) Ciencias Químicas, 10\% (50/500) Economía, 19\% (95/500) Ingeniería, 11.8\% (59/500) Filosofía y Letras, 39.6\% (198/500) Derecho y 3.60\% (18/500) Matemáticas. La edad promedio de los estudiantes fue de $21.29 \pm 3.13$ años, con un rango de 17 a 44 años.

Cuadro 1.

Coeficiente alfa de Cronbach de la escala de hiperestrés.

\begin{tabular}{lc}
\hline Sub-escala & alfa \\
\hline Estilo de vida & .550 \\
Ambiente & .771 \\
Síntomas & .811 \\
Empleo/ocupación & .625 \\
Relaciones & .646 \\
Personalidad & .746 \\
Total escala & .911 \\
\hline
\end{tabular}

\section{Prevalencia de estrés}

La prevalencia de hiperestrés de los estudiantes de las seis Unidades Académicas fue de $44.4 \%$ (estrés elevado + estrés peligroso), la mayoría de los estudiantes $(47.8 \%)$ presentó estrés normal; estrés peligrosamente bajo sólo el $1.4 \%$, estrés bajo $6.4 \%$, estrés elevado $30 \%$ y estrés peligroso $14.4 \%$.

En el porcentaje de hiperestrés en cada una de las Unidades Académicas, el mayor porcentaje lo tuvo Ciencias Químico-Biológicas (56\%), en segundo lugar Filosofía y Letras (52.5\%), continuando con Derecho (48.9\%), Economía (47.8\%), Ingeniería (41.9\%) y el menor porcentaje Matemáticas (20\%).

\section{Nivel de estrés por semestre}

El mayor porcentaje de estrés elevado se presentó en el quinto semestre con un $40 \%$ (38/95) y en el séptimo semestre con $31.25 \%$ (40/128); mientras que para estrés peligroso fue el tercer semestre con $19.44 \%$ (14/72) y el séptimo semestre con $17.19 \%$ (22/128) (Cuadro 2).

\section{Cuadro 2.}

Nivel de estrés en relación a los semestres en estudiantes universitarios.

\begin{tabular}{lccccc}
\hline & \multicolumn{5}{c}{ Semestre } \\
Nivel de Estrés & $\begin{array}{c}\text { Primero } \\
\%\end{array}$ & $\begin{array}{c}\text { Tercero } \\
\text { Quinto }\end{array}$ & $\begin{array}{c}\text { Séptimo } \\
\%\end{array}$ & $\begin{array}{c}\text { Noveno } \\
\%\end{array}$ \\
\hline $\begin{array}{l}\text { Peligrosamente } \\
\text { Bajo }\end{array}$ & 2.08 & 1.39 & 2.11 & 0.78 & 0 \\
Bajo & 6.94 & 5.56 & 2.11 & 7.81 & 9.84 \\
Normal & 50.70 & 48.6 & 43.15 & 42.97 & 57.38 \\
Elevado & 27.08 & 25.0 & 40.0 & 31.25 & 24.59 \\
Peligroso & 13.19 & 19.44 & 12.63 & 17.19 & 8.19 \\
TOTAL ALUMNOS & 144 & 72 & 95 & 128 & 61 \\
\hline
\end{tabular}

\section{Nivel de estrés por turno}

El $55.20 \%(276 / 500)$ es del turno matutino y el $44.80 \%$ (224/500) del turno vespertino. Los alumnos del turno vespertino presentaron mayores niveles de estrés elevado y peligroso en relación a los del turno matutino (Cuadro 3). 
Cuadro 3.

Porcentaje del nivel de estrés por turno.

\begin{tabular}{lcc}
\hline Nivel de Estrés & Matutino & Vespertino \\
\hline Peligrosamente Bajo & 1.1 & 1.8 \\
Bajo & 6.5 & 6.3 \\
Normal & 52.2 & 42.4 \\
Elevado & 28.6 & 31.7 \\
Peligroso & 11.6 & 17.9 \\
\hline
\end{tabular}

\section{Nivel de estrés por sexo}

El 52.8\% (264/500) pertenece al sexo femenino y el $47.2 \%$ (236/500) al sexo masculino. El 54.9\% $(145 / 264)$ de las mujeres presentó hiperestrés y el $39.77 \%$ (105/264) estrés normal. El 32.63\% (77/236) de los estudiantes varones presentó hiperestrés y $56.78 \%$ (134/236) tuvo estrés normal.

\section{Nivel de estrés y estado civil}

En relación al estado civil, el 89.60\% (448/500) refirió ser soltero, el $7.6 \%$ (38/500) casado, el $0.8 \%$ (4/500) divorciado y el 2\% (10/500) unión libre. El $30.58 \%$ (137/448) de los solteros presentó estrés elevado y el $13.62 \%$ (61/448) estrés peligroso. El 26.32\% (10/38) de los casados presentó estrés elevado y el $18.42 \%$ (7/38) presentó estrés peligroso. De los cuatro estudiantes divorciados, uno presentó estrés elevado y un estudiante presentó estrés peligroso. De los que viven en unión libre, dos presentaron estrés elevado $\mathrm{y}$ tres presentaron estrés peligroso.

\section{Presión arterial}

El 85\% (425/500) presentó presión arterial normal, el 12.60\% (63/500) presión arterial alta y sólo el 2.40\% (12/500) presión arterial baja. De los 63 estudiantes que tuvieron presión arterial alta (mayor de 130/90 $\mathrm{mmHg}$ ), el 47.61\% (30/63) presentó hiperestrés. La media de la presión sistólica fue de 116.28 $\pm 12.1 \mathrm{DE}$ con un rango de 76 a $151 \mathrm{mmHg}$, y de la presión diastólica fue de $72 \pm 11.05 \mathrm{DE}$ con un rango de 42 a $117 \mathrm{mmHg}$.

\section{Pulso}

El 5.2\% (26/500) presentó pulso acelerado (taquicardia), el $87.4 \%$ (437/500) pulso normal y sólo el $7.4 \%$ (37/500) pulso lento (bradicardia).

\section{Cuadro 4.}

Medidas antropométricas de los estudiantes universitarios de la ciudad de Chilpancingo, Guerrero.

\begin{tabular}{|c|c|c|}
\hline & Media & Valor mínimo y máximo \\
\hline Estatura & $1.61 \pm .09$ & 1.37 a $1.87 \mathrm{~m}$. \\
\hline Peso & $63.53 \pm 14.18$ & 34 a $145 \mathrm{~kg}$. \\
\hline $\begin{array}{l}\text { Índice de masa } \\
\text { corporal }\end{array}$ & $24.30 \pm 4.15$ & 15.52 a $46.28 \mathrm{~kg} / \mathrm{m} 2$ \\
\hline $\begin{array}{l}\text { Circunferencia } \\
\text { cintura }\end{array}$ & $82.78 \pm 10.49$ & 57 a $134 \mathrm{~cm}$. \\
\hline
\end{tabular}

El 59.40\% (297/500) tuvo un IMC normal, el $26.40 \%$ (132/500) sobrepeso y el $9.40 \%$ (47/500) obesidad. Los estudiantes con sobrepeso y diagnóstico de hiperestrés suma el 46.21\% (61/132), y los que están obesos y con hiperestrés el $44.68 \%$ (21/47). Siendo estos estudiantes los más propensos a sufrir hipertensión.

\section{Circunferencia cintura $(C C)$}

De los estudiantes varones, el 80.08\% (189/236) tuvo las medidas de cintura normal, el 9.75\% (23/236) una cintura igual o mayor a $94 \mathrm{~cm}$, y de éstos el $34.78 \%$ (8/23) tuvo diagnóstico de hiperestrés y el $10.17 \%$ (24/236) presentó la medida de la cintura igual o mayor a $102 \mathrm{~cm}$, de éstos el 29.16\% (7/24) tuvo diagnóstico de hiperestrés.

El 51.89\% (137/264) de mujeres estudiantes presentó las medidas de cintura normal, el $28.79 \%$ (76/264) presentó una cintura igual o mayor a $80 \mathrm{~cm}$ y de éstas el $59.21 \%$ (45/76) tuvo diagnóstico de hiperestrés. El $19.32 \%$ (51/264) presentó la medida de cintura igual o mayor a $88 \mathrm{~cm}$ y de éstas el $62.74 \%$ (32/51) alcanzó diagnóstico de hiperestrés.

\section{Índice Cintura Cadera (ICC)}

En los hombres, el promedio del índice cinturacadera (ICC) fue de $0.86 \pm 0.05$; esto indica que el promedio de los estudiantes tiene un bajo riesgo de padecer un problema de salud relacionado con la obesidad. 


\section{Cuadro 5.}

Variables que mostraron asociación con el hiperestrés en el Modelo de Regresión Logística.

\begin{tabular}{lccc}
\hline VARIABLE & OR & IC 95\% & Valor p \\
\hline No hacer ejercicio & 4.13 & $1.90-8.94$ & 0.000 \\
Consumo de alcohol & 6.41 & $2.62-15.66$ & 0.000 \\
Vivir en espacios reducidos & 5.19 & $2.43-11.12$ & 0.000 \\
No relajarse en casa & 6.87 & $2.98-15.79$ & 0.000 \\
Dormitorio pequeño & 3.86 & $1.86-8.01$ & 0.000 \\
Sensación de ahogo & 7.46 & $2.47-22.46$ & 0.000 \\
Cansado y sin energía & 3.45 & $1.69-7.03$ & 0.001 \\
Sudar sin realizar actividad física & 4.28 & $1.83-9.99$ & 0.001 \\
Llorar y desesperarse con facilidad & 3.63 & $1.70-7.72$ & 0.001 \\
Ocupaciones que no permiten comer tranquilamente en casa. & 6.86 & $3.31-14.20$ & 0.000 \\
Llevarse mal con los compañeros de trabajo o escuela. & 6.32 & $2.16-18.52$ & 0.001 \\
No tener buena relación con el jefe (padre, profesor). & 7.28 & $2.74-19.33$ & 0.000 \\
Exaltarse al discutir & 4.27 & $1.94-9.41$ & 0.000 \\
Pensar en lo que se va a decir cuando se discute. & 3.28 & $1.56-6.89$ & 0.002 \\
No tener confianza en el futuro & 5.59 & $2.19-14.22$ & $1.98-9.25$ \\
Pensar en lo peor & 4.28 & $1.39-6.65$ & 0.000 \\
Pensar en los que le deben dinero & 3.04 & 0.000
\end{tabular}

El 4.24\% (10/236) de los estudiantes varones presentó un ICC mayor de 0.95 , y el $2.12 \%$ (5/236) de éstos padecen hiperestrés. En las mujeres el promedio del índice cintura-cadera (ICC) fue de $0.81 \pm 0.05$; esto indica que el promedio de las estudiantes tienen un bajo riesgo de padecer un problema de salud relacionado con la obesidad. El 26.89\% (71/264) de las mujeres presentó un ICC mayor de 0.85 , y el 13.64\% (36/264) de ellas padece de hiperestrés.

\section{Análisis multivariado}

Se hizo el análisis bivariado, y las variables que individualmente tuvieron un nivel de significancia estadística (<0.05) se incluyeron en el modelo de Regresión Logística. Las variables que salieron asociadas al hiperestrés se muestran en el Cuadro 5.

\section{Discusión}

Este estudio permitió conocer los niveles de estrés en la población de estudiantes de la Ciudad Universitaria la de Chilpancingo, Guerrero, y se logró observar cómo el estrés afecta a la población estudiantil sin importar qué licenciatura cursen, edad, sexo y estado civil, asimismo se identificaron los agentes estresantes que se asocian al hiperestrés.

La población estudiada presentó las siguientes prevalencias: estrés peligroso $14.40 \%$ y estrés elevado $30 \%$. Estas prevalencias fueron superiores a las reportadas por Maruris et al. (2011), que fueron del 10.03\% estrés peligroso y $24.27 \%$ estrés elevado. La diferencia se debe tal vez al tipo de población estudiada, ya que Maruris trabajó con población abierta y nuestra muestra fue de estudiantes.

Las unidades académicas con un mayor número de estudiantes con hiperestrés fueron Ciencias Químico Biológicas con un 56\% y Filosofía y Letras con un $52.54 \%$.

Las carreras del área de la salud, como Medicina, Odontología, Enfermería, Estomatología y Psicología, están consideradas como escuelas muy demandantes académicamente y se han reportado mayores niveles de estrés en los estudiantes que cursan estas licenciaturas (Bedoya, Perea \& Ormeño, 2006; Amat et al., 2006; Caldera \& Pulido,2007; Pulido, et. al., 2011; Bermúdez et al., 2006). En el presente estudio, el mayor nivel de estrés lo tuvieron los estudiantes de Ciencias Químico Biológicas, también del área de la salud, lo cual confirma los hallazgos de los anteriores autores. Sin embargo, también se encontraron altos niveles de estrés en los alumnos de Filosofía y Letras, que no corresponde al 
área de la salud, por lo tanto no sólo los estudiantes del área de la salud se estresan, sino también de otras áreas del conocimiento. De esto se deduce que los estudiantes se estresan por el exceso de trabajo académico y presiones de otro tipo (económicas). En el caso de Filosofía y Letras, los alumnos refirieron estresarse mucho por no tener un horario fijo y tal vez porque algunos de los estudiantes trabajan. En el caso de Ciencias Químico Biológicas, los estudiantes tienen sobrecarga de trabajo académico y presiones políticas por los constantes cambios de las autoridades universitarias (cambio de Director y Rector), pues algunos profesores condicionan las calificaciones al cambio del voto.

Los estudiantes menos estresados fueron los de la Unidad Académica de Matemáticas con $20 \%$ de hiperestrés, esto se debe posiblemente a que el número de alumnos en cada grupo es reducido y los profesores les brindan una mejor atención.

Un estudio realizado por Díaz et al. (2008), encontró en un grupo de estudiantes de Odontología, una mayor prevalencia de estrés elevado en el séptimo semestre del 22\% ; y en nuestro estudio se encontró un mayor porcentaje de estrés elevado en estudiantes del quinto semestre $(40 \%)$ y en el séptimo semestre (31.25\%). Cabe señalar que en Ciencias Químicas, en el séptimo semestre los estudiantes tienen una sobrecarga de trabajo, debido a que realizan su servicio social y prácticas profesionales, además de que las materias que llevan en este semestre son muy demandantes. Otros investigadores también han hallado mayores niveles de estrés en el primero, séptimo y noveno semestre en estudiantes de Medicina (Restrepo, Jaramillo \& Marín, 1993).

Un estudio realizado por Bermúdez et al. (2006) en estudiantes de Medicina, encontró una asociación entre el consumo de alcohol y el estrés elevado; en este estudio también encontramos esta asociación con un $\mathrm{OR}=6.41$, IC95\% 2.62-15.66 у p= 0.000 (Bermúdez et al., 2006).

Las mujeres presentaron $54.92 \%$ de hiperestrés comparado con el estudio que realizó Caldera, Pulido y Martínez (2007), que fue de 19.39\% de estrés elevado, es decir, la prevalencia fue más elevada en nuestro estudio. Aún así, en otros estudios las mujeres presentan mayor nivel de estrés que los hombres (Barra, Cerna, Kramm \& Véliz, 2006); sin embargo, los resultados en relación al estrés en estudiantes de dos universidades difieren según el tipo de universidad. En los estudiantes de universidad pública hubo diferencias significativas por sexo de los estudiantes (las mujeres presentaron mayor puntaje que los hombres), pero no en la universidad privada (González \& Landero, 2006).
Lo anterior puede deberse a las diferentes condiciones sociales y escolares.

Maruris et al. (2011) encontraron una asociación entre hiperestrés y las personas que lloran y se desesperan con facilidad. En esta investigación, esta variable también se encontró asociada $(\mathrm{OR}=3.39$, IC95\% 2.32-6.89 у p = 0.001). Los estudiantes del sexo masculino con una medida de cintura igual o mayor a $94 \mathrm{~cm}$ y con diagnóstico de hiperestrés fue de $31.91 \%$ (15/47) y las del sexo femenino que tuvieron una medida de cintura igual o mayor a $80 \mathrm{~cm}$ y con diagnóstico de hiperestrés fue del 60.63\% (77/127); todos estos estudiantes tienen un riesgo mayor de sufrir complicaciones metabólicas relacionadas con la obesidad; como lo mencionan algunos médicos del Instituto Mexicano del Seguro Social, la grasa que se encuentra alrededor de los órganos y en su interior producen sustancias nocivas que alteran la presión arterial, sumado a esto el estilo de vida acelerado que tiene la población estudiantil, la presión de trabajo, el estrés de la ciudad y la falta de tiempo para llevar una alimentación sana (IMSS Puebla, 2010).

En cuanto al promedio de la presión arterial sistólica, fue de $116.28 \pm 12.1 \mathrm{mmHg}$ y la diastólica de $72 \pm$ 11.05; estas presiones fueron mayores a las reportadas por Campo y colaboradores, donde la sistólica fue de $106.4 \pm 10.5 \mathrm{mmHg}$ y la diastólica $69.7 \pm 8.8 \mathrm{mmHg}$ (Campo, Reyes, Ortiz \& Herrera, 2006).

Cabe mencionar que los estudiantes varones presentaron mayor prevalencia de presión arterial alta con el $69.84 \%$ (44/63), comparado con las mujeres que fue de $30.16 \%$ (19/63). Morales y colaboradores reportan una asociación entre sexo masculino y la hipertensión arterial, hallando que los jóvenes tienen más de tres veces el riesgo de sufrir hipertensión arterial, en relación a las mujeres. Por lo cual se recomienda realizar ejercicio para disminuir el riesgo de padecer hipertensión (Morales, Corro, Angulo \& Zavala, 2003).

En este estudio, el Indice de Cintura Cadera (ICC) de riesgo para la obesidad visceral fue mayor en las mujeres con un $26.89 \%$ (71/264), en relación a los hombres que fue de 4.24\% (10/236). Montalbán Sánchez (2001), en un estudio realizado en 85 sujetos de Málaga, reporta un índice de cintura cadera en mujeres de 68\% y en hombres de $34.3 \%$, es decir, también fue mayor el porcentaje en el sexo femenino.

En esta investigación se observa una alta prevalencia de hiperestrés en los estudiantes no solamente del área de la Salud, sino también del área Social, por lo que es necesario atender este problema brindando apoyo psicológico, instalando un módulo de atención para los 
estudiantes en Ciudad Universitaria de Chilpancingo, Guerrero, México.

\section{Conclusiones}

De los 17 factores estresantes asociados al hiperestrés se encuentran: sensaciones de ahogo, no tener buena relación con el jefe (padre, profesor), no relajarse con tranquilidad en casa, ocupaciones que no permiten comer tranquilamente en casa, consumo de alcohol, llevarse mal con los compañeros, no hacer ejercicio y sentirse cansado y sin energías, entre otros.

De acuerdo a la alta prevalencia de estrés que presentan los estudiantes de las Unidades Académicas de Ciencias Químico-Biológicas y Filosofía y Letras, se recomienda realizar una exhaustiva investigación en dichas unidades para conocer cuál es el problema que aqueja a los estudiantes y el apoyo de un psicólogo para mejorar la salud mental de los estudiantes, ya que el nivel de estrés puede depender de la evaluación emocional o cognitiva de cada persona. Además, sería interesante evaluar otras variables que puedan explicar las diferencias encontradas entre sexos.

\section{Referencias}

Amat, V., Fernández, C., Orts, I., Poveda, M., Romá, M. y Rivera, D. (2006). Estrés en estudiantes de enfermería. Rev ROL de Enfermería, 133, 75-78.

Barra, E., Cerna, R., Kramm, D. y Véliz, V. (2006). Problemas de salud, estrés, afrontamiento, depresión y apoyo social en adolescentes. Terapia Psicológica, 24 (1), 55-61.

Barraza, A. (2004). El estrés académico en los alumnos de postgrado. Extraído el 23 de abril del 2009 desde http://www.psicologiacientifica.com/publicaciones/biblioteca/articulos/ar- barraza02-3.htm.

Barraza, A. (2008). El estrés académico en alumnos de maestría y sus variables moduladoras: un diseño de diferencia de grupos. Avances en Psicología Latinoamericana, 26 (2), 270-289.

Bedoya, S., Perea, M., y Ormeño, R. (2006). Evaluación de niveles, situaciones generadoras y manifestaciones de estrés académico en alumnos de tercer y cuarto año de una Facultad de Estomatología. Rev Estomatol Herediana, 16 (1), 15-20.

Bermúdez, S., Duran, M., Escobar, C., Morales, A., Monroy, S., Ramírez, A., Ramírez, J., Trejos, J., Castaño, J. y González, S. (2006). Evaluación de la relación entre rendimiento académico y estrés en estudiantes de Medicina. Rev Med UNAB, 9, 198-205.

Caldera, J., Pulido, B. y Martínez, M. (2007). Niveles de estrés y rendimiento académico en estudiantes de la carrera de Psicología del Centro Universitario de Los Altos. Rev de Educación y Desarrollo, 7, 77-82.

Campo, J., Reyes, J., Ortiz, C. y Herrera, J. (2006). Niveles de la presión arterial y de estrés psicosocial en estudiantes de la Facultad de Salud, Universidad del Valle. Cali, Colombia, 2003-2004. Rev Colomb Med, 37 (2), 21-25.

Carmel, S. y Bernstein, J. (1987). Perceptions of Medical School Stressors: their Relations hip to Ago, Year of study and Trait Anxiety, Journal Human Stress, 13 (1), 39-44.

Díaz, D., Portugal, J., Roa, A., Romero, G. T., Salgado, S., et al. (2008). Nivel de estrés en estudiantes de la carrera de odontología de la FESI en el ciclo escolar 2008-1. Extraído el 21 de abril del 2009 desde http://www.google.com.mx/search?hl=es\&g=nivel+ de+estres+en+estudiants+de+la+carrera+de+odontolog\%C3ADa + de+la+fesi+en+el+ciclo+escolar+2008-1\&meta $=$.

González, M. y Landero, R. (2006). Síntomas psicosomáticos y estrés en estudiantes de Psicología. Revista de Psicología Social. 21, (2), 141-152.

Hernández-Mendoza, E., Cerezo-Reséndiz, S. y López-Sandoval, M. (2007). Estrategias de afrontamiento ante el estrés laboral en enfermeras. Rev Enferm Inst Mex Segur Soc., 15 (3), 161-166.

Maruris, M., Cortés, P., Gómez, L. y Godínez, F. (2011). Nivel de estrés en una población del sur de México. Psicología y Salud, $21,(2), 229-244$.

Melgosa, J. (2006). ¡Sin estrés!. Ed. Safeliz: Madrid, España. pp.18-33.

Montalbán, J. (2001). Índice cintura/cadera, obesidad y estimación del riesgo cardiovascular en un centro de salud de Málaga. Medicina de familia (AND), 2 (3), 208-215.

Morales, J., Corro M., Angulo, B. y Zavala, F. (2003). Hipertensión arterial. Riesgo para los jóvenes. Revista de Investigación en salud, 5 (2). Extraído el 6 de junio del 2010 desde http://redalyc.uaemex. $\mathrm{mx} / \mathrm{redalyc} / \mathrm{src} / \mathrm{inicio} /$ ArtPdfRed.jsp?iCve $=14250204$.

Polo, A., Hernández, J. M. y Pozo, C. (1996). Evaluación del estrés académico en estudiantes universitarios. Ansiedad y estrés, 2 $(2,3), 159-172$.

Restrepo, A., Jaramillo, F. y Martin, J. (1993). Estrés en estudiantes de Medicina del Instituto de Ciencias de la Salud. Revista CES Medicina, 2 (1), 38-43.

Román, C., Ortíz, F. y Hernández, Y. (2008). El estrés académico en estudiantes latinoamericanos de la carrera de Medicina. Revista Iberoamericana de Educación, 46 (7), 1-8. 
